\title{
The Establishment of Three-Dimensional Model of Human Knee Joint
}

\author{
Ziyue Liu, Fuzhong Wang \\ Department of Physics, Tianjin Polytechnic University, Tianjin, China \\ Email: liufred@live.cn
}

Received 13 December 2014; revised 28 December 2014; accepted 6 January 2015

Copyright (C) 2015 by authors and Scientific Research Publishing Inc.

This work is licensed under the Creative Commons Attribution International License (CC BY). http://creativecommons.org/licenses/by/4.0/

(c) () Open Access

\begin{abstract}
Objective: To discuss a method to establish a three-dimensional model of healthy human knee joint, which can be used for further knee joint biomechanics analysis and simulation. Methods: CT scan and medical image three-dimensional reconstruction software (Mimics) were used to obtain the knee joint three-dimensional finite element model (FEM) according to reverse engineering theory. Results: FEM of knee joint with complete bone structure was established by Mimics. Conclusion: Three-dimensional FEM was established according to CT images exports as IGES file. The model can be used for knee joint biomechanics finite element analysis to provide references and proposals for the clinical diagnoses of knee joint illness, and the design of artificial knee joint prosthesis.
\end{abstract}

\section{Keywords}

\section{Knee Joint, Mimics, Three-Dimensional Model}

\section{Introduction}

With the weight-bearing and motor function, knee joint is the most complex joint in human body. It is easily hurt or caught various diseases due to the mechanical environment and movement condition it is located. With the fastest-ageing society on earth, osteoarthritis and other diseases related will be a hot spot and it will affect people's life standard. Reinforcing the research capacity has a magnificent meaning for improving elder people's living quality. Current studies consider that the mechanism of osteoarthritis no matter caused by sports injuries or cartilage failure is the improper stress distribution in the joint [1]-[4]. It is hard to explain the mechanism of stress transfer and distribution by traditional biomechanics research. With the assistance of finite element analysis, we can stimulate movement of joint in various conditions, and by this way we can acknowledge the stress distribution in different parts. To establish a relatively complete finite element model of the health human knee joint with proper mechanical properties and anatomy structure is the basis for further re- 
search and provides a digital experiment platform for repeated stimulation. In this paper, we introduce a method to establish three-dimensional finite element model of human knee joint based on CT images and conduct a simple analysis on the model to verify its accuracy. The main procedure of our research is shown in Figure 1 . This process is somewhat like 3-D printing technology that both are the procedure of 3-D model reestablishment. While 3-D printing is to rebuild a real object according to the digital data, the model established in this essay is stored as electronic document available for knee joint finite element analysis.

\section{Materials and Methods}

\subsection{Devices and Software}

Computer (CPU: Intel ${ }^{\circledR}$ Core $^{\mathrm{TM}}$ i5-2430 2.40 GHz*2, RAM: 8.00 GB, GPU: GT-540M, OS: win7), CT images, Mimics medical image three-dimensional rebuilding software, Ansys 11.0 finite element analysis software. The CT images come from the database of visible human project of the US national library of medicine. $8001 \mathrm{~mm}$ scanned healthy woman lower limbs CT images were downloaded as Dicom format. Mimics was programmed in 1992 and widely used in digital medical field. The initial propose was to apply CT scan images in rapid prototyping manufacturing. With the growing improvement, it is now widely used in Computer-Aided Mechanical Engineering, including medical three-dimensional modeling based on medical images, computer-aided design, finite element and hydromechanics analysis, rapid prototyping manufacturing, visual operation planning, anthropotomy measurement analysis, etc. Ansys is a powerful engineering stimulation software widely used in many fields including structural mechanics, multiphysics, fluid dynamics, explicit dynamics, electromagnetics, and hydrodynamics.

\subsection{Experiment Method}

1) Input the data of the model: import the 800 Dicom CT images to Mimics. Original axial view, coronal plane and vertical plane from the scanned data was observed as Figure 2.

2) Rebuild the three-dimensional model: image thresholding segmentation was the first step of the modeling procedure and mask was obtained. Since it is the knee joint that need modeling, we selected the object region by "region growing" in the toolbar, shown as Figure 3, distal femur, patella, tibial plateau of the right leg was selected. The default gray value of the bone is only reference, the auto generated mask may not cover all the bone in the region. There would be a lot of small cracks, bumps and pits, shown as Figure 4. We need to modify the mask until all the area is covered completely. At last "smooth object" option was used to optimize the model produced, the knee joint model is shown as Figure 5.

3) Preliminary treatment of the finite element analysis: before the analysis, the model needed to be meshed to finite element grid model, this was obtained by FEA module in Mimics. The grid was optimized by remesh module. The finite element model (Figure 6) was obtained for further research.

4) Apply material properties to the model:

The parameters in Table 1 were preferred from previous literature [5].

5) Loads and boundary conditions: approximately $40 \%$ of the body weight is applied to each knee when standing. In this paper, we consume the total weight is $60 \mathrm{Kg}$, so $235 \mathrm{~N}$ was applied on the mechanical axis of

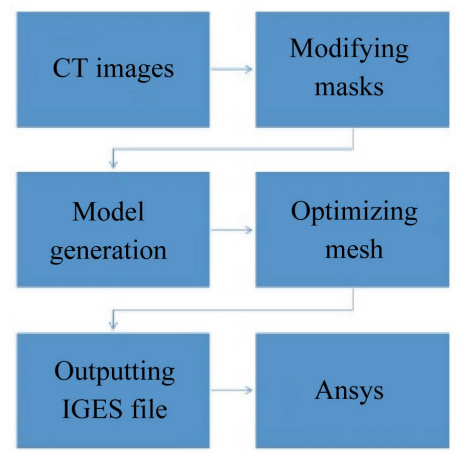

Figure 1. Research procedure. 


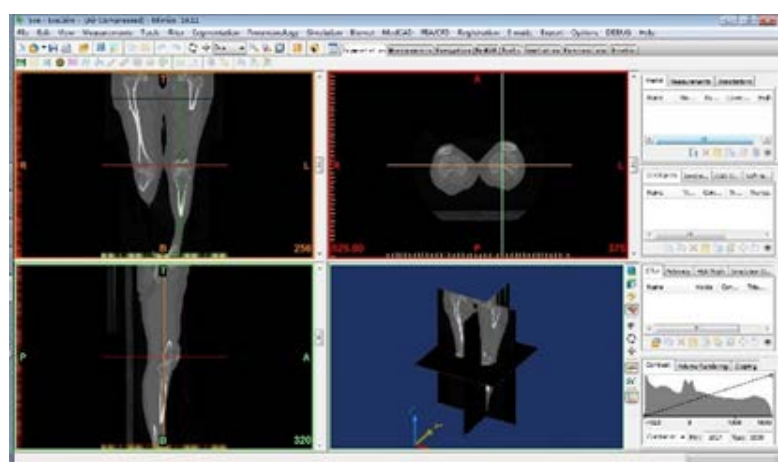

Figure 2. Lower limbs tomoscan.

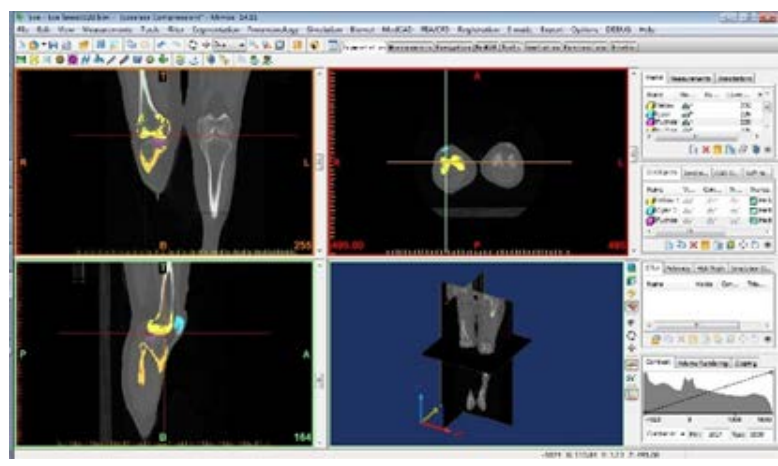

Figure 3. Region selected.

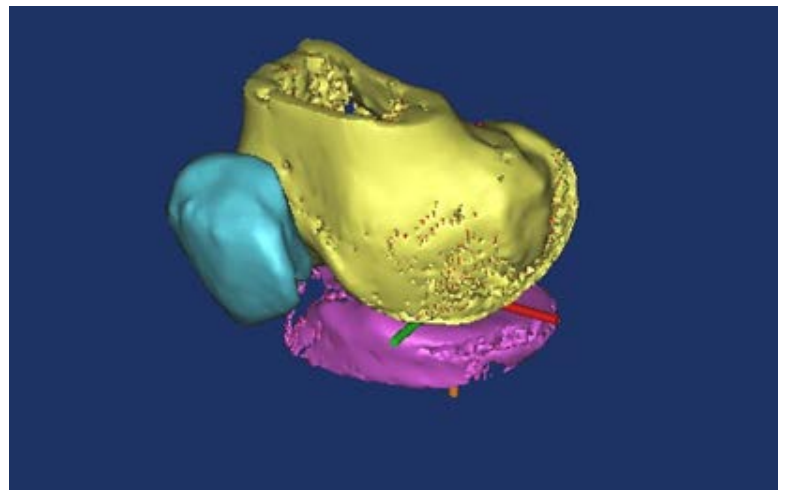

Figure 4. Flaw of the original model.

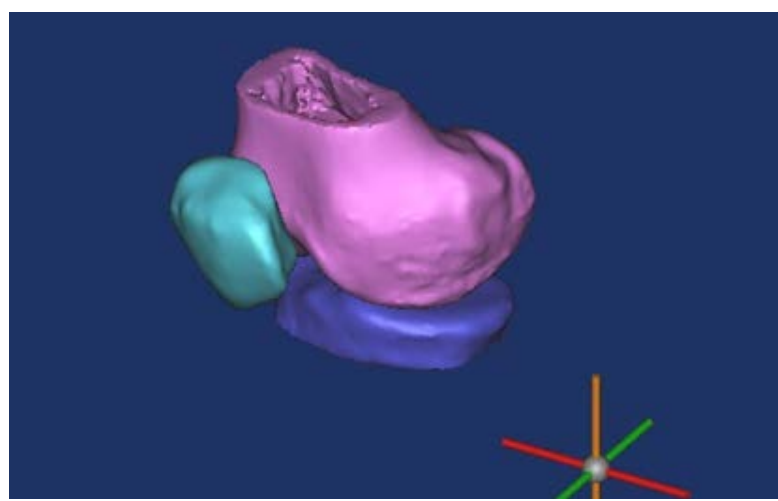

Figure 5. Model after optimization. 


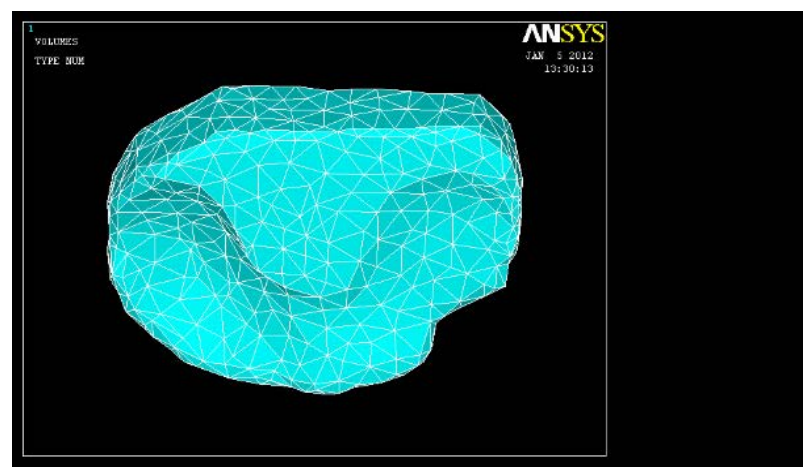

Figure 6. The gridded tibial plateau.

Table 1. Material properties of the components of the knee joint model.

\begin{tabular}{ccc}
\hline Material & Young's modulus $(\mathrm{MPa})$ & Poisson's radio $(\mu)$ \\
Femur & 12,000 & 0.3 \\
Tibia & 6900 & 0.49 \\
Patella & 12,000 & 0.3 \\
\hline
\end{tabular}

the top femur. The boundary condition was set that tibia and femur were kept fixed in all direction. The model was solved by Ansys 11.0.

\section{Results}

In this paper, we established the model of a healthy human knee joint, and conducted biomechanics analysis to it. Figure 7 shows the Von Mises stress distribution of the tibial plateau.

Von Mises stress was chosen to evaluate the stress index in this analysis. It is a combined stress defined according to the $4^{\text {th }}$ strength theory reflecting the average stress level each dot inside the material, and one of the most objective indices in finite element analysis [5]. The maximum of the Mises stress locates in the contact region of femoral condyle and tibial plateau. The maximum stress is $25 \mathrm{MPa}$ at the edge of the tibial plateau. The result is corresponded with clinical cases that injury of inner side meniscus and tibial plateau is more common and the maximum of the Von Mises stress accord with other researches [6]. This means that the model established can be used to present the authentic status of human knee joint.

\section{Discussion}

In this research, we built the knee joint model including distal femur, patella, and tibial plateau precisely based on the CT images of human lower limbs. This method can be used to establish knee joint model fast and accurately, and the file is suitable to directly transfer to finite element analysis software (Ansys) for biomechanics simulation.

However, the soft tissue of the knee joint (ligament, joint capsule, synovial fluid, etc.) was not obtained. This is due to the fact that CT image cannot provide the soft tissue outline as clear as the bone tissue [7]. To accomplish this part, one way is to depict the soft tissue according to the anatomy structure of knee joint on the CTconducted model which may contribute to the untruthfulness of the model because of the lack of accuracy. Another method is to use MRI image which can show soft tissue clearly [8]. We will integrate our present work with MRI image in the future.

Model with intact knee joint structure can be used in the further finite element analysis to simulate knee joint under certain conditions and the information obtained is beneficial to knee arthropathy diagnosis and knee prosthesis design [9]. The advantages of finite element analysis are evident that the model can be tested constantly, the cost is lower than traditional research, and most importantly it can reveal the inner interaction of knee joint such as the stress distribution of the surface of the tibial plateau in different gaits. These are all problems that 


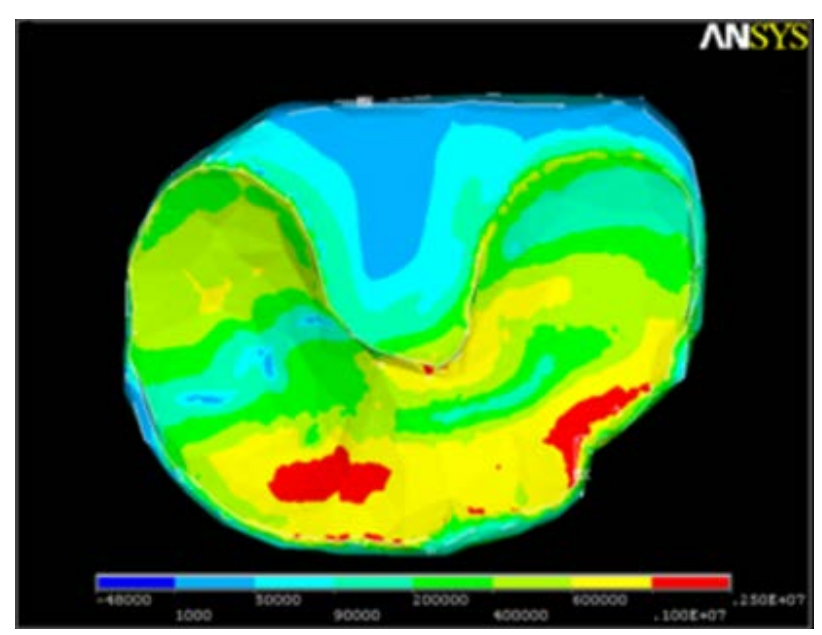

Figure 7. The Von Mises stress distribution of the tibial plateau.

traditional specimen test and clinical research cannot solve. Since 1960s, finite element method has widely applied in biomechanics analysis. It had successfully utilized in hip joint, knee joint, spine, shoulder, etc. [10]. The finite element analysis is a strong addition and useful tool in the study of biomechanics and it will become the development trend in the future.

\section{References}

[1] Zhang, S.N. and Lu, A.Y. (2005) Animal Model of Musculoskeletal Motion Injury Research. Chinese Journal of Sports Medicine, 31, 185-188.

[2] Zhang, H.P. and Song, J.R. (2010) Establishment and Application of Animal Experimental Models of Acute Skeletal Muscle Injury. Journal of Clinical Rehabilitative Tissue Engineering Research, 11, 458-463.

[3] Zhao, T., Weng, L., You, Y.-H., et al. (2013) The Appearance of X-Ray and MR Imaging in Osteochondral Fracture of Knee Joint after Acute Injury. Chinese Journal of Radiology, 37, 985-988.

[4] Teng, Y., Wang, Z., Li, D.C., et al. (2008) Fabrication of Custom-Made Artificial Semi-Knee Joint Based on Rapid Prototyping Technique: Three-Dimensional Reconstruction of Femoral Condyle. Chinese Journal of Reparative and Reconstructive Surgery, 41, 257-260.

[5] Niu, Y.F. (2012) The Impact of Cartilage Hardening of the Knee Articular Cartilage to the Stress Distribution of Knee Joint. M.Sc. Thesis, Tianjin Polytechnic University, Tianjin.

[6] Chantarapanich, N., Nanakorn, P., Chernchujit, B., et al. (2009) A Finite Element Study of Stress Distributions in Normal and Osteoarthritic Knee Joints. Journal of the Medical Association of Thailand, 17, 223-226.

[7] Gíslason, M.K., Stansfield, B. and Nash, D.H. (2010) Finite Element Model Creation and Stability Considerations of Complex Biological Articulation: The Human Wrist Joint. Medical Engineering and Physics, 34, 246-249.

[8] Luring, C., Hufner, T., Perlick, L., et al. (2006) The Effectiveness of Sequential Medial Soft Tissue Release on Coronal Alignment in Total Knee Arthroplasty: Using a Computer Navigation Model. Journal of Arthroplasty, 21, 428-434. http://dx.doi.org/10.1016/j.arth.2005.05.031

[9] Manda, K., Ryd, L. and Eriksson, A. (2011) Finite Element Simulations of a Focal Knee Resurfacing Implant Applied to Localized Cartilage Defects in a Sheep Model. Journal of Biomechanics, 44, 794-801. http://dx.doi.org/10.1016/j.jbiomech.2010.12.026

[10] Hopkins, A.R., New, A.M., Rodriguez-y-Baena, F., et al. (2010) Finite Element Analysis of Unicompartmental Knee Arthroplasty. Medical Engineering and Physics, 15, 347-351. 
Scientific Research Publishing (SCIRP) is one of the largest Open Access journal publishers. It is currently publishing more than 200 open access, online, peer-reviewed journals covering a wide range of academic disciplines. SCIRP serves the worldwide academic communities and contributes to the progress and application of science with its publication.

Other selected journals from SCIRP are listed as below. Submit your manuscript to us via either submit@scirp.org or Online Submission Portal.
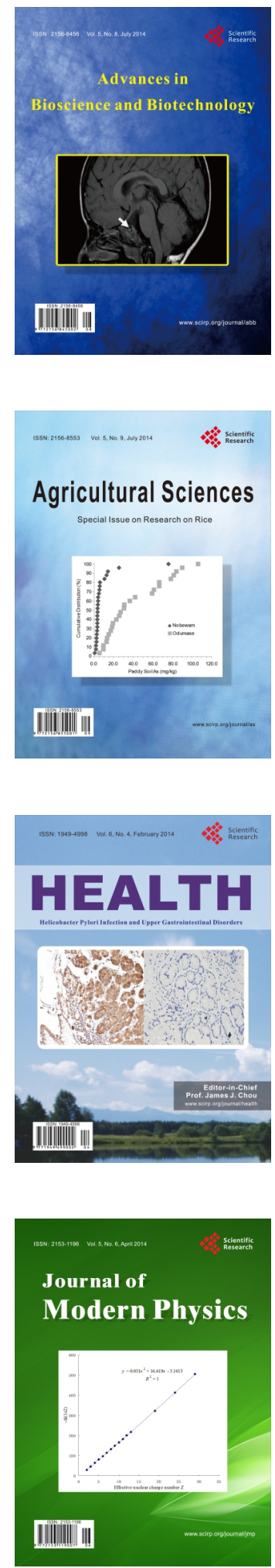
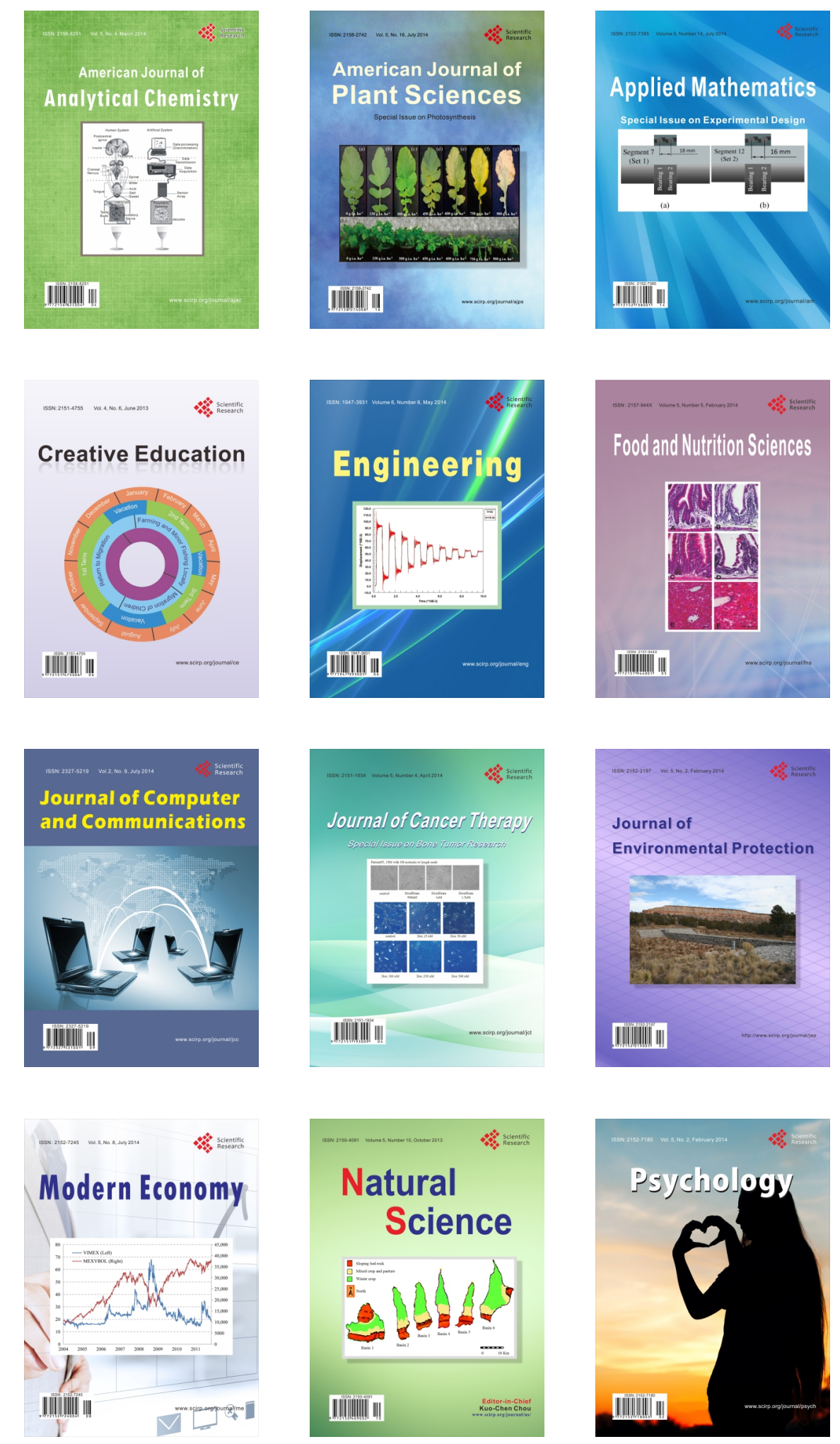